\title{
ESA uplink modeling of satellite earth systems
}

\author{
Timur A. Zubov \\ Magistracy student \\ Siberian Federal University \\ Krasnoyarsk, Russian Federation \\ timonische@bk.ru
}

\author{
Vitaly V. Sukhotin \\ Phd in Engineering, Associate professor \\ Siberian Federal University \\ Krasnoyarsk, Russian Federation \\ vsuhotin@sfu-kras.ru
}

This paper describes the uplink structure of ESA standards and modeling of the structure without noise adding and RFblocks. SES

Key words: simulation, modulation, BPSK, FM, Uplink, ESA,

\section{INTRODUCTION}

At present time there are many data exchange approved standards and technologies. The myriad requirements of the standards and its design complexity without signal illustrative example in the time and frequency domains complicate study, familiarization and performance evaluation of operation system. In this connection, objective of the article is only the forming of the appearance model of ESA uplink using MATLAB Simulink with Communications System Toolbox. In more detail of Evaluation parameters ESA uplink you may familiarize in [1]. The toolbox algorithms includes of channel coding, modulation, MIMO and OFDM and also it supports $\mathrm{C}$ or HDL code generation [1].

\section{STRUCTURE DESCRIPTION}

Modeling structure is based on ETSI EN 301926 [3], approved European Space Agency. This standard describes stages for standard modulation, it is illustrated on fig. 1. At first digital data of the Data Source block are encoded by the Packet Encoder block (block code, interleave). The packet encoder output data may be encoded by the FEC Encoder. After that an encoded data are step-by-step modulated including the following stages: pulse code modulating (PCM), binary phase shift keying (BPSK) and analog phase or frequency modulating.

The uplink standard modes for data exchange between earth and satellite station (SES) is defined in fig. 1

TABLE I. MODULATION STANDARD MODES AND POTENTIAL CONFIGURATION

\begin{tabular}{|l|c|}
\hline \multicolumn{1}{|c|}{$\begin{array}{c}\text { Communication } \\
\text { direction }\end{array}$} & Standard Mode \\
\hline Uplink & $\begin{array}{c}\text { TeleCommand mode (TCM1): } \\
\text { PCM/BPSK/FM }\end{array}$ \\
\hline Downlink & TeleMetry mode (MTM1): PCM/BPSK/PM \\
\hline
\end{tabular}

Article considers modulation processing of the TeleCommand mode (TCM1). There is the following modulation requirements of the TCM1 mode.

\section{A. Pulse code modulation}

PCM of TCM1 mode may be the bipolar non-return-to-zero level (NRZ-L) or the bipolar non-return-to-zero mark (NRZM). Signal rate shall be limited between 8 symbols/s up to 4000 symbols/s.

UPLINK TX

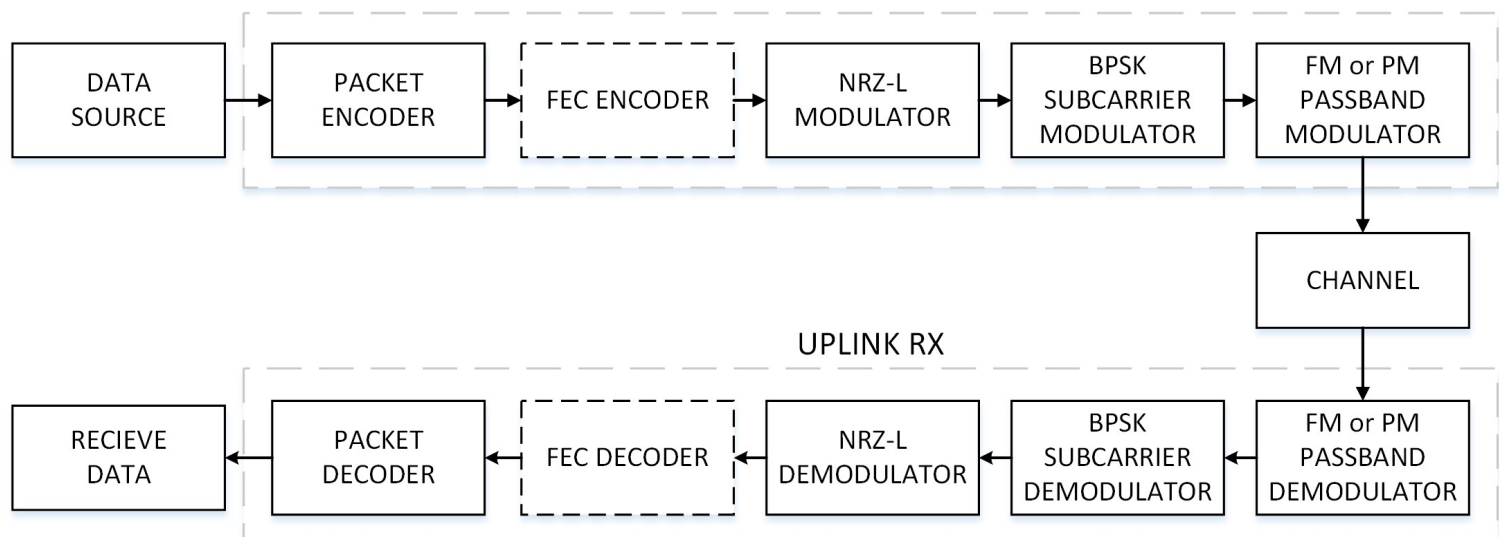

Fig. 1. The satellites earth stations (SES) standard mode uplink structure of ETSI EN 301926 reference 


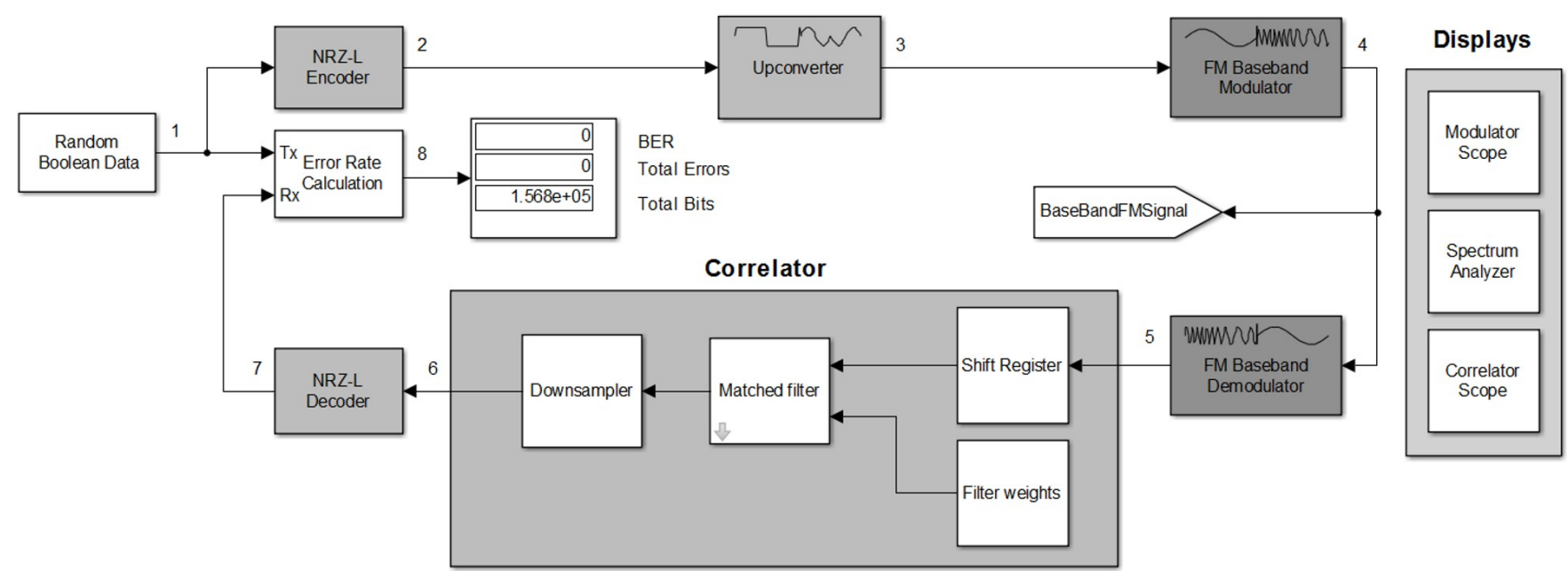

Fig. 2. The SES standard mode uplink modeling structure of ETSI EN 301926 reference

\section{B. Binary phase shift keying}

The PCM output signal is the modulating signal for BPSK. The subcarrier frequency shall be $8 \mathrm{kHz}$ for all telecommand rates up to $2000 \mathrm{sym} / \mathrm{s}$. A $16 \mathrm{kHz}$ subcarrier shall be used only in cases where the $4000 \mathrm{sym} / \mathrm{s}$ symbol rate is needed or when required by the operator.

\section{Analog frequency modulation}

A FM signal is modulated by a BPSK subcarrier signal. The deviation frequency shall be limited up to $\pm 400 \mathrm{kHz}$.

\section{UPLINK MODELING}

The uplink model bases on structure shown on fig. 1. In general it consist of data source, uplink modulator, uplink demodulator and displays, that is illustrate on fig. 2. There are not channel coding blocks in the model to consider modulation processing. The Random Boolean Data forms random Boolean stream with 2000 Boolean symbol/s.

\section{A. Signal modulating}

the bit stream is transformed to a sequence polar pulse waveforms [4] by the NRZ-L Encoder. The NRZ-L pulse curves are illustrate on fig. 3 and fig. 4 as the PCM.

The phase of the subcarrier waveform with is shifted to one of two states by the modulating NRZ-L signal in the Upconverter in the time domain. In other words baseband NRZ-L signal is shifted up to the subcarrier frequency, in the frequency domain [5]. The subcarrier frequency in this example is $4 \mathrm{kHz}$ for demonstrate its curves are shown on fig. 3 and fig. 4 as the BPSK.

The FM Baseband Modulator generates a complex baseband frequency modulated signal from a BPSK subcarrier waveform. A baseband FM signal differs from standard frequency modulated passband signal [6] then it is derived from the passband representation by downcovertioning it by carrier frequency. Removing the component at double carrier frequency result leaves the baseband signal representation [7]:

$$
S_{F M}(t)=\frac{A}{2} \exp \left[j 2 \pi f_{\Delta} \int_{0}^{t} s_{B P S K}(\tau) d \tau\right]
$$

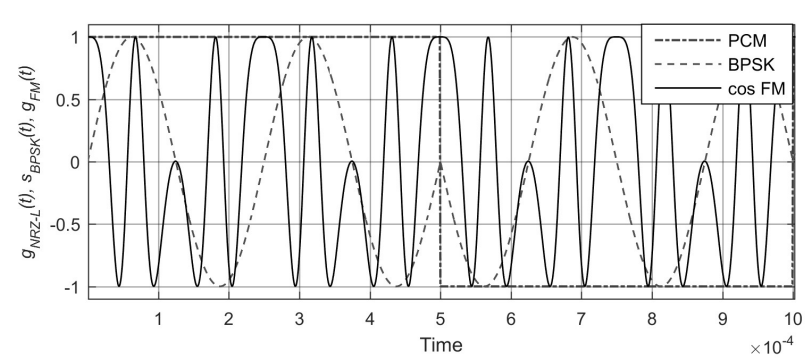

Fig. 3. Time diagram of signal modulating is step-by-step divided into three step: PCM, BPSK, FM inphase component

As can be seen from the inphase (illustrated on fig. 3 as the cos. FM) and quadrature (illustrated on fig. 4 as the sin. FM) curves its shape is distorted by unlimited spectrum aliasing of image channels. To shift up a baseband FM signal to passband frequency it should be multiplied by a radiofrequency complex signal and the result inphase and quadrature components are should be summed.

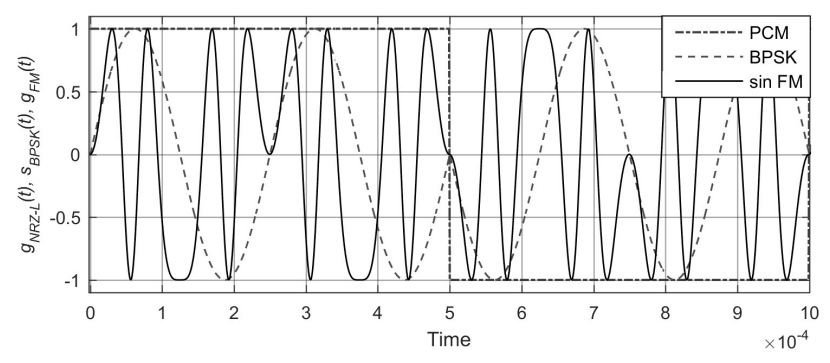

Fig. 4. Time diagram of signal modulating is step-by-step divided into three step: PCM, BPSK, FM quadrature component

The Baseband FM and subcarriear BPSK signal spectrums are shown on fig. 5. 


\section{B. Signal demodulating}

Demodulating in the uplink receiver of the Satellite station as modulating in the uplink transmitter of the Earth station is processed step-by-step. A received signal waveform is demodulated by The FM demodulator. The correlator shall be used to regenerate a digital signal from a demodulated BPSK waveform by the FM demodulator. There is not a bandpass correlation detector in The Communications System Toolbox.

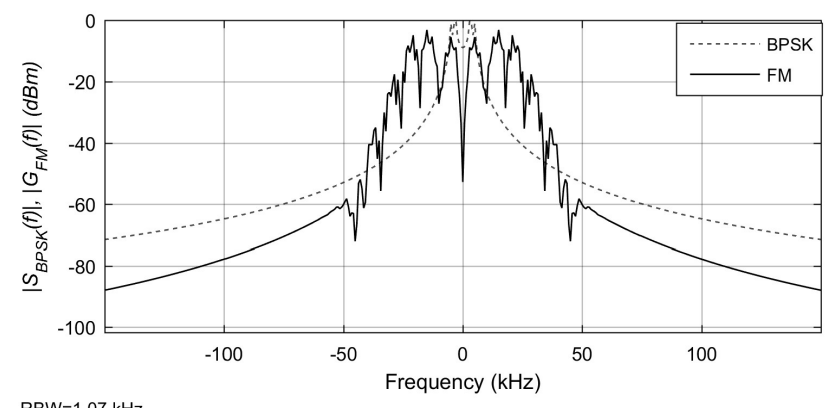

Fig. 6. The Baseband FM and subcarriear BPSK signal spectrums

\section{Correlation detecting of a received signal}

The correlation detecting of received signal is divide into three steps:

- A received continuous-time and continuous-amplitude BPSK signal $r(t)$ is converted to a discrete-amplitude sequence of samples $r(k)$ with a sampling rate $f$ s. At the times of $t=k / f \mathrm{~s}$, the samples are shifted into the register so that earlier samples are located to the right of later samples. Each time first sample of the each symbol has been gotten, the shift register is reset to design correct matched filter.

- The Filter weight is a buffer storing filter weights or coefficients of the prototype waveform $c(n)$, where $n=$ $0, \ldots, N-1$ is the time index of weights and register stages.

- At the times of $t=k / f \mathrm{~s}$, the Matched Filter multiplies the shift register and the filter weights by element and sums result. In other words, the matched filter operates the discrete form of the convolution integral [8]:

$$
z(k)=\sum_{n=0}^{N-1} r(k-n) c(n)
$$

- The downsampler decreases the sample rate to symbol rate and results the last sample of the matched filter sequence corresponding end of the symbol period.

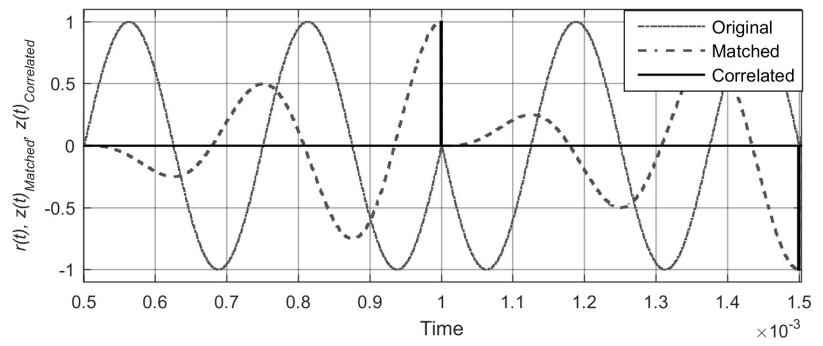

Fig. 7. The time diagram of signal correlating is step-by-step divided into three step: reciveing, matched filtering, downsampling

The NRZ-L decoder is a block converts a NRZ-L pulse sequence into a bit stream.

A regenerated received digital signal should be validated by comparing it with bitstream from the Data Source. A Received and transmitted data is compared by the Error Rate Calculation which results a bit-error rate value, a total errors value and a total bits value. The results of validation is shown on fig. 2 .

\section{CONCLUSION}

The paper results the simulation uplink model of ETSI EN 301926 standard mode and the processing time and spectrum diagram. The uplink model differs from the uplink structure then there are not a channel coding and RF blocks in the model. The model processing is realized on baseband frequency. In next time a noise and distorting effects influence and a system operating in radio-frequency region will be considered in model.

\section{REFERENCES}

[1] «Evaluation of BER for BPSK modulation and FM modulated by $B P S K »$, T. A. Zubov, V. V. Sukhotin Achievements of Modern Radioelectronics, №10, 2015.

[2] Communications System Toolbox. Getting Started Guide. The MathWorks, Inc., 2011-2015.

[3] Draft ETSI EN 301926 V1.1.1 Reference. Satellites Earth Stations and Systems (SES); Radio Frequency and Modulation Standard for Telemetry, Command and Ranging (TCR) of Geostationary Communications Satellites. European Telecommunications Standards Institute, 2001.

[4] Galkin V.A. Cifrovaja mobil'naja radiosvjaz'. Uchebnoe posobie dlja vuzov. M.: Grjachaja linija - Telekom, 2007, 432s.

[5] Feer K. Besprovodnaja cifrovaja svjaz'. Metody moduljacii i rasshirenija spektra. M.: Radio i svjaz', 2000. 520s.

[6] Kolosovskij E.A. Ustrojstva priema i obrabotki signalov. Uchebnoe posobie dlja vuzov. M: Gorjachaja linija - Telekom, 2007. 456s.

[7] Communication Systems Toolbox. Reference. The MathWorks, Inc., 2011-2015.

[8] Bernard Sklar. Digital communication. Fundamentals and applications. Prentice-Hall PTR, New Jarsey. 2007. 